\title{
Fondements et pratique de l'alternance en formation à l'enseignement professionnel : quelques données de recherches autour de l'élaboration et de la mise en œuvre à l’Université de Sherbrooke
}

\section{Claudia Gagnon, Élisabeth Mazalon et Anne Rousseau}

Volume 13, numéro 1, 2010

L'entrée en enseignement professionnel : images d'une réalité et questions de formation

URI : https://id.erudit.org/iderudit/1017459ar

DOI : https://doi.org/10.7202/1017459ar

Aller au sommaire du numéro

Éditeur(s)

Faculté d'éducation, Université de Sherbrooke

ISSN

1911-8805 (numérique)

Découvrir la revue

Citer cet article

Gagnon, C., Mazalon, É. \& Rousseau, A. (2010). Fondements et pratique de l'alternance en formation à l'enseignement professionnel : quelques données de recherches autour de l'élaboration et de la mise en œuvre à l'Université de Sherbrooke. Nouveaux cahiers de la recherche en éducation, 13(1), 21-41. https://doi.org/10.7202/1017459ar
Résumé de l'article

Cet article décrit, dans un premier temps, le contexte particulier de la formation des enseignants en enseignement professionnel au Québec. Il expose ensuite quelques fondements autour de la notion d'alternance et précise les choix qui ont été faits pour la conception du programme de baccalauréat en enseignement professionnel (BEP) de l'Université de Sherbrooke. À partir de données issues de focus group et de questionnaires, réalisés auprès des étudiants et de divers intervenants au cours de la mise en œuvre, ce texte questionne, dans un troisième temps, l'alternance comme mode d'organisation de la formation et comme stratégie pédagogique, et se termine par une discussion autour de l'ingénierie de l'alternance dans le contexte du BEP et des enjeux qui y sont liés. 


\title{
Fondements et pratique de l'alternance en formation à l'enseignement professionnel : quelques données de recherches autour de l'élaboration et de la mise en œuvre à l'Université de Sherbrooke
}

\author{
Claudia Gagnon, Élisabeth Mazalon et Anne Rousseau \\ Université de Sherbrooke
}

\section{Résumé}

Cet article décrit, dans un premier temps, le contexte particulier de la formation des enseignants en enseignement professionnel au Québec. Il expose ensuite quelques fondements autour de la notion d'alternance et précise les choix qui ont été faits pour la conception du programme de baccalauréat en enseignement professionnel (BEP) de l'Université de Sherbrooke. À partir de données issues de focus group et de questionnaires, réalisés auprès des étudiants et de divers intervenants au cours de la mise en œuvre, ce texte questionne, dans un troisième temps, l'alternance comme mode d'organisation de la formation et comme stratégie pédagogique, et se termine par une discussion autour de l'ingénierie de l'alternance dans le contexte du BEP et des enjeux qui y sont liés.

\section{Abstract}

This article first describes the context specific to the training of vocational-education teachers in Quebec. It then presents certain basic issues related to the concept of work-study and explains the choices that were made in designing the program of the Universite de Sherbrooke's Bachelor's in Vocational Education (BVE). Then, based on data obtained from focus-group sessions and questionnaires conducted with students and various involved persons during the program's implementation, the article questions the work-study approach as a way of organizing training and as a pedagogic strategy. The article concludes with a discussion of the design of work-study in the BVE context and of the issues associated with it. 


\section{Introduction}

Les programmes de formation des enseignants ont fait l'objet d'une révision majeure au cours des dernières années au Québec et le secteur de l'enseignement professionnel n'y a pas échappé. Fondés sur une approche par compétences et dans une perspective de professionnalisation et d'approche culturelle de l'enseignement (Gouvernement du Québec, 2001a), ces nouveaux programmes accordent une importance accrue à la formation en milieu de pratique. À l'Université de Sherbrooke, le programme de formation des enseignants en enseignement professionnel a été conçu dans une logique de formation continue des étudiants/enseignants, avec la volonté d'ancrer la formation dans la pratique de l'enseignement, de réaffirmer l'importance du métier initial dans le parcours de formation, et de répondre aux besoins exprimés et aux réalités spécifiques de la clientèle concernée. Cet article devrait interpeler particulièrement les chercheurs en sciences de l'éducation et dans d'autres champs disciplinaires intéressés par des modèles pédagogiques intégrateurs, de même que les directions de centres de formation professionnelle désireuses de mieux comprendre l'articulation de la démarche de qualification pédagogique de leur personnel enseignant.

\section{Poser le problème de la formation en enseignement professionnel au Québec}

\subsection{Une histoire récente}

Au Québec, les enseignants de la formation professionnelle s'appuient uniquement sur leur formation technique et sur leur expérience de travail pour transmettre leurs savoirs (Charland, 1982; Hardy, Desrosiers-Sabbath et Defrênes, 1995), et ce, jusqu'aux années 1960, où des programmes de formation en pédagogie commencent à voir le jour (Charland, 1982). Au début des années 1970, un certificat de 30 crédits permet aux enseignants déjà en poste de bénéficier d'un perfectionnement psychopédagogique. En 1975, le ministère de l'Éducation du Québec (MEQ) invite les universités à offrir, en plus du perfectionnement psychopédagogique de 30 crédits nécessaires à l'obtention du permis ou du brevet d'enseignement, un perfectionnement dans le métier et de culture personnelle de 60 crédits, créant ainsi le programme de baccalauréat de 90 crédits, mais les enseignants du secteur reprochent «aux universités de donner un programme trop théorique et de ne pas être en mesure de leur proposer des cours appropriés à leurs besoins particuliers » (Gouvernement du Québec, 2001a, p. 14-15). Quoi qu'il en soit, et malgré les différents avis pointant du doigt la nécessaire revalorisation de la formation professionnelle et le manque de système efficace de formation des enseignants de ce secteur (Caron, 1997; Comité d'orientation de la formation du personnel enseignant, 1998; Conseil supérieur de l'éducation, 1984, 1992; Gouvernement du Québec, 2001a; Groupe de travail sur la relance de la formation professionnelle des jeunes au secondaire et de la formation technique, 1995), le certificat de premier cycle demeure la norme.

En 2001, dans la perspective d'offrir aux enseignants du professionnel une formation plus adéquate et comparable à celle des enseignants des autres secteurs de formation, le MEQ dépose une nouvelle version de programme menant à l'obtention d'un diplôme de baccalauréat, diplôme qui devient nécessaire pour obtenir la licence d'enseignement (temporaire) après 90 crédits de formation et le brevet d'enseignement (permanent) au terme du programme (120 crédits). Ce programme de formation s'adresse aux personnes qui ont cumulé 4500 heures d'expérience dans le 
métier, qui sont déjà en poste à titre d'enseignants dans les centres de formation professionnelle ou qui désirent se préparer à l'enseignement (Gouvernement du Québec, 2001a). Il devient un passage obligé pour obtenir une autorisation légale d'enseigner et un contrat d'enseignement à temps plein ou partiel.

\subsection{Une clientèle et un contexte particuliers}

Contrairement aux autres secteurs de formation des enseignants où les étudiants doivent terminer leur baccalauréat avant d'accéder à un poste en enseignement, 95\% des étudiants en enseignement professionnel occupent dans le même temps un poste d'enseignant dans un centre de formation professionnelle, où ils ont été recrutés sur la base de leur expertise de métier (Balleux, 2003 ; Balleux et Loignon, 2004; Balleux, 2006a). La pression institutionnelle et gouvernementale concernant cette obligation de formation est amplement ressentie par les enseignants en poste, qui se voient souvent forcés de retourner - bien malgré eux - sur les bancs d'école, alors que pour plusieurs, le défi est de taille considérant que leur âge moyen est de 42,1 ans au moment de leur engagement (Tardif, 2001) ${ }^{1}$ et que les compétences du métier ont été souvent acquises par la pratique davantage que par de longues études (Comité d'orientation de la formation du personnel enseignant, 1998). Pour une grande partie d'entre eux, il y a passage du niveau secondaire à l'université, plusieurs années après avoir quitté les bancs d'école (Beaucher et Gagnon, 2008), et ce passage difficile est malheureusement souvent empreint de beaucoup de tension et de frustration chez les enseignants de métiers (Balleux, 2006b).

\subsection{Un problème de développement de programme de formation adapté}

En somme, les particularités des étudiants, tout comme le contexte dans lequel ils évoluent, posent le problème de la formation à l'enseignement professionnel, notamment en termes :

- d'adhésion des enseignants à la formation, ce qui renvoie au défi de concevoir un programme de BEP innovant, adapté à leurs besoins et qui tienne compte de leur réalité (Beaucher, Chatigny, Balleux, Tardif et Gagnon, 2005);

- de structure ou d'organisation de la formation, en envisageant le programme dans une perspective de formation continue en cours d'emploi bien qu'il s'agisse d'une formation initiale;

- de nécessité de penser un accompagnement pédagogique des étudiants, tant dans les établissements de formation professionnelle qu'à l'université, étant donné leur faible scolarisation, pour la plupart;

- de réponse à un cadre de référence ministériel qui doit se déployer dans différents contextes d'enseignement, ce qui signifie qu'il faille concevoir un programme de formation qui soit à la fois souple, en permettant des parcours individualisés et en s'adaptant aux différents métiers et aux particularités d'enseignement de ces métiers, et rigide, en respectant les règles du ministère de l'Éducation, du Loisir et du Sport (MELS) et en visant le développement des 12 compétences en enseignement.

1 Lâge moyen du personnel enseignant permanent en formation professionnelle est le plus élevé du système scolaire québécois, soit 50 ans et plus (Gouvernement du Québec, 1999). 
L'élément majeur de cette réforme de la formation des enseignants en enseignement professionnel et dans d'autres secteurs a été, sans contredit, l'intégration de stages d'une durée significative dans la formation (Gouvernement du Québec, 2001a). La formation en milieu de pratique impliquant une gestion différente et une valorisation des ressources des différents milieux, cette nouvelle façon d'entrevoir la formation à l'enseignement a constitué, pour l'équipe du BEP de l'Université de Sherbrooke, l'élément déclencheur pour introduire un nouveau modèle de formation basé sur l'ingénierie de l'alternance, articulant les activités de formation en milieu scolaire et en milieu universitaire, un modèle de formation en alternance qui allait au-delà de la simple juxtaposition des stages et du modèle organisationnel. Ceci étant dit, comment développer un programme de baccalauréat en enseignement professionnel basé sur l'ingénierie de l'alternance qui réponde à la fois aux besoins et aux réalités des étudiants, ainsi qu'aux exigences ministérielles en regard du développement des compétences en enseignement? Comment articuler les périodes de formation à l'université à la pratique professionnelle dans les établissements de formation professionnelle, pour des étudiants adultes en cours d'emploi?

Dans un premier temps, il nous a semblé important d'effectuer une analyse critique des écrits théoriques et des recherches portant sur l'alternance en vue de fonder nos choix organisationnels et pédagogiques en regard du développement du programme de formation.

\section{Définir les fondements organisationnels et pédagogiques: repères théoriques de l'alternance en formation}

«L'alternance en sciences de l'éducation procède d'une succession de périodes de travail ou de vie active en entreprise, et de périodes d'études dans un établissement de formation s'inscrivant dans un rapport théorie-pratique» (Bourgeon, 1979, p. 11). Alors que certains considèrent l'alternance de façon générale comme une approche (Audet, 1995) ou un courant pédagogique (Danvers, 1992), d'autres y voient plutôt, sans les opposer, un mode d'organisation de la formation (Mathey-Pierre, 1998; Gouvernement du Québec, 2001b) ou une formule éducative, une stratégie pédagogique (Gouvernement du Québec, 2001b) ou de formation.

Outre les définitions qui mettent en relief l'articulation entre les deux lieux de formation ou qui renvoient davantage à l'arrimage théorie-pratique, de multiples appellations ont été données au terme alternance: alternance cognitive (Lerbet-Séréni et Violet, 1999; Morandi, 1999), alternance formative (Morandi, 1999), alternance juxtapositive (Bourgeon, 1979; Geay, 1999), alternance intégrative (Dumont et Wilk, 1992), alternance qualifiante (Dumont et Wilk, 1992), alternance socioformative (Galvani, 1999), alternance approchée (Bachelard, 1994), alternance tripolaire (Denoyel, 1999), pour n'en nommer que quelques-unes. (Gagnon, 2007, p. 145)

Dans la perspective d'une typologie basée sur l'arrimage des pratiques éducatives (incluant les objets, les processus et les éléments organisationnels de la formation) (Gagnon, 2007, 2008), trois principaux types d'alternance ont été retenus; ils sont repris ici en tentant de les adapter au contexte de formation à l'enseignement professionnel.

Le type juxtaposition - qui réfère à des périodes de travail comme enseignant dans un établissement de formation professionnelle et des périodes de formation à l'université qui sont sans liaison entre elles. 
Le type complémentarité - qui réfère à la répartition de la formation entre l'établissement de formation professionnelle et l'université selon les spécificités de chacune.

Le type intégration - qui réfère à une formation globale au sein de l'établissement de formation professionnelle et de l'université.

Quoi qu'il en soit, l'alternance est un concept élastique (Bachelard, 1994), une notion polymorphe (Agulhon, 2000) qui recouvre aujourd'hui de nombreuses pratiques (Gimonet, 1998; Voisin, 1993). En formation à l'enseignement, l'usage du mot alternance est assez récent et s'inscrit dans une vision globale de professionnalisation (Gagnon et Mazalon, 2010a, 2010b; Maubant, 2007 ; Vanhulle, Merhan et Ronveaux, 2007; Wittorski, 2007).

\subsection{L'alternance comme mode d'organisation de la formation à l'enseignement professionnel}

Comme mode d'organisation de la formation, l'alternance se distingue de la formation traditionnelle, qui se déroule uniquement dans un milieu scolaire comme l'université, par cinq dimensions : l'espace, le temps (la durée et le rythme), les ressources, les relations et les modalités pédagogiques (Mazalon et Landry, 1998). Les prochaines sous-sections abordent les quatre premières dimensions, tandis que certaines modalités pédagogiques seront détaillées à la section suivante.

\subsubsection{L'espace: entre université, milieu scolaire et milieu professionnel}

Si plusieurs auteurs ont pensé l'alternance comme le va-et-vient entre le milieu de formation et l'entreprise ou le milieu de travail (Bachelard, 1994; Doray et Fusulier, 2002; Fusulier, 2001 ; Hardy, 2003 ; Jedliczka et Delahaye, 1994; Malglaive et Weber, 1983; Schneider, 1999), dans le cadre du BEP, il faut plutôt voir l'alternance entre trois lieux, trois organisations, trois cultures. En effet, alors que la plus grande partie du programme se déroule en alternance entre l'université et l'établissement de formation professionnelle où l'étudiant enseigne, il n'est pas rare que l'étudiant qui débute en enseignement poursuive en même temps ses activités professionnelles en entreprise, étant donné la précarité d'emploi en enseignement à laquelle il fait face (Balleux, 2006b). Les exigences ministérielles visant aussi le perfectionnement dans le métier enseigné, certaines activités de formation pourraient également se dérouler dans les milieux professionnels. Ainsi, au cours de sa formation, l'étudiant est confronté à trois univers culturels avec leurs valeurs, leur propre conception du savoir et leur propre langage (Tilman et Delvaux, 2000), trois univers qui peuvent être sensiblement différents, voire se contredire (Dumont et Wilk, 1992). Quoique différents, ces trois lieux doivent être envisagés à la fois dans leur spécificité et dans leur complémentarité (Fusulier, 2001), comme un seul «espace de formation», et l'étudiant doit pouvoir identifier et reconnaitre la valeur de ce qu'il peut attendre de chacun des lieux (Gagnon, 2008). Suivant Engeström et Kärkkäinen (1995), c'est à travers les conflits et difficultés vécus au sein des différents espaces de formation que les apprenants sont appelés à construire leurs savoirs. Pour que s'opère un véritable boundary crossing, traduit par Vanhulle, Merhan et Ronveaux (2007) comme «une traversée optimale d'un espace à l'autre» (p. 15), il importe que ces passages entre les différents espaces soient soutenus par des outils de formation et l'implication de différents acteurs partenaires (Engeström, Y., Engeström, R. et Kärkkäinen, 1995), sur lesquels nous reviendrons plus loin. 


\subsubsection{Le temps: durées et rythmes de l'alternance}

Envisager l'alternance entre trois lieux de formation, c'est également considérer trois rythmes de fonctionnement ou trois rapports au temps: le temps professionnel, rythmé en fonction de la productivité; le temps scolaire, supposé rythmé en fonction de l'apprentissage des élèves, mais suivant plutôt la structure régulière et contraignante des périodes de cours qui s'enchainent les unes après les autres de façon uniforme et linéaire, que ce soit de jour ou de soir, des programmes de formation professionnelle divisés en modules, et parfois de l'alternance entre le centre de formation et l'entreprise ou le milieu de travail (Husti, 1983, dans Savoie-Zajc, 2001; Tardif et Lessard, 1999); et le temps universitaire, divisé en sessions et en fins de semaine de formation, suivant un rythme qui est supposé requis pour effectuer les apprentissages en enseignement. Pour les enseignants qui débutent dans l'établissement de formation professionnelle avant même de commencer leur formation en enseignement, ils se trouvent «face à un rythme d'action effréné qui le[s] pousse[nt] à agir dans l'urgence et l'incertitude» (Reviol, 2001, p. 183). «Cette première "formation pratique" qui, dans l'urgence et la survie ressemble à de la formation sur le tas, avec peu de support en général et peu d'encadrement, les place d'emblée dans une formation à deux vitesses» (Balleux, 2006b, p. 610).

Considérant ces différents rythmes de fonctionnement ou ces rapports au temps auxquels l'étudiant est confronté, l'alternance proposée dans le cadre du BEP doit être modulable. À cet égard, Savoie-Zajc (2001) a examiné la dimension temporelle qui affecte la mise en place de l'alternance en formation professionnelle à partir de la grammaire temporelle proposée par McGrath et Kelly (1992, dans Savoie-Zajc, 2001) qui comprend cinq paramètres : la fréquence, la durée, la périodicité, la séquence, la localisation temporelle. Ces derniers paramètres peuvent aider la réflexion qui entoure la mise en place de l'alternance dans la formation à l'enseignement professionnel.

\subsubsection{Les relations: importance des acteurs dans l'alternance}

Dans le contexte des stages et de l'alternance dans la formation à l'enseignement, la majorité des auteurs parlent de la triade constituée de l'étudiant, du superviseur universitaire et de l'enseignant associé, également appelé maitre de stage dans le milieu scolaire (Arpin et Capra, 2008; Baillauquès, Lavoie, Chaix et Hétu, 2002; Boutet et Pharand, 2008; Boutet et Rousseau, 2002; Boutin, 2002; Correa Molina et Gervais, 2008; Correa Molina, Gervais et Rittershaussen, 2008; Gervais, 1995; Gervais et Desrosiers, 2005 ; Martin, Garant, Gervais et St-Jarre, 2000; Portelance et Tremblay, 2006; Tardif et Ziarko, 1997). Dans la plupart des cas, les auteurs font fi des titulaires des cours à l'université, ce qui laisse sous-entendre une certaine scission entre les stages et les cours qui, à notre avis, n'a pas sa place dans une réelle alternance.

L'alternant ${ }^{2}$, jonction paradoxale de l'élève à l'école et de l'apprenti en entreprise, ou de l'étudiant à l'université et de l'enseignant novice dans l'établissement de formation professionnelle, doit être perçu comme l'acteur central de sa formation, car c'est lui seul qui vit concrètement ce va-et-vient entre les différents espaces de formation (Chaix, 1993; Geay, 1994; Geay, 1999; Mathey-Pierre, 1998; Mazalon et Bourassa, 2003; Mazalon, Beaucher et Langlois, 2010; Tilman et Delvaux, 2000). Il est donc amené à se construire une identité spécifique (ses propres représentations) et à construire son propre savoir. Ainsi, pour certains, l'alternance renvoie-t-elle à une

2 Terme de plus en plus utilisé pour désigner l'apprenant en alternance (Clénet, 2002; Landry et Mazalon, 2002). 
position constructiviste de l'apprentissage, voire socioconstructiviste (Candau et Kittel, 2001; Clénet, 2002; Geay et Salaberry, 1999; Gérard, 2000; Tilman et Delvaux, 2000), où le métier d'apprenant est transformé.

Pour Lerbet-Séréni et Violet (1999), en formation professionnelle, si les processus paradoxaux que l'alternance met en jeu sont des points d'appui aux apprentissages signifiants chez l'apprenant, ils amènent à penser le rôle de l'enseignant, que l'on pourrait attribuer au titulaire du cours et au superviseur universitaire dans notre cas, comme un rôle de médiation en vue de soutenir le passage entre théorie et pratique, entre pratique et théorie, entre la formation à l'université et la formation dans le milieu scolaire, ou entre le travail prescrit et le travail réel. Il doit conduire le processus (Agulhon, 2000) en se préoccupant de la cohérence de la formation, faisant le lien entre la logique d'apprentissage dans l'établissement de formation professionnelle et celle vécue à l'université, dans le but de favoriser l'émergence et la construction de sens chez l'alternant (Galvani, 1999).

\subsubsection{Les ressources matérielles: des outils indispensables}

Que ce soit en formation à l'enseignement ou dans d'autres types de formation, différents auteurs ont traité de l'importance des ressources matérielles dans l'alternance ou des outils de l'alternance pour soutenir les différents acteurs dans le processus de formation (Bachelard, 1994; Boënnec et Maurel-Indart, 2001 ; Engeström et al, 1995; Gomez et Hostein, 1997; Landry, 1992; Lerbet-Séréni et Violet, 1999; Schneider, 1999; Tilman et Delvaux, 2000; Violet, 1997). Ces outils concernent à la fois les documents administratifs tels les documents d'information et les protocoles d'entente ou contrats entre les organisations partenaires, et les documents pédagogiques : journal de bord (De Cock, 2001), guide de cheminement, guide de stage, portfolio, mémoire de formation professionnelle, rapport de stage (Merhan, et Baudouin, 2007), outil internet comme le forum électronique (Boutin 2002). Bien que peu de recherches exposent les aspects positifs et les limites de l'utilisation de l'un ou l'autre de ces outils, tous semblent considérer la présence d'outils comme étant indispensable à la formation en alternance.

\subsection{L'alternance comme stratégie pédagogique de la formation à l'enseignement professionnel}

À l'instar de Mathey-Pierre (1998), nous notons que «la prise en compte du potentiel formateur des situations de travail et son articulation interactive avec les situations de formation sont parmi les caractéristiques essentielles de l'alternance» (p. 72). Ainsi, en tant que stratégie pédagogique, l'alternance constitue un va-et-vient nécessaire à la connaissance pour "permettre au savoir formalisé de se confronter à la pratique et au savoir pratique de se formaliser» (Aballea, 1991, p. 11). En ce sens, elle implique un processus itératif des activités d'apprentissage dans les différents espaces permettant aux apprenants de prendre conscience du savoir professionnel (disciplinaire et psychopédagogique) à acquérir dans un contexte d'enseignement. À ce sujet, la définition de Meirieu (1992), en ce qui a trait à l'alternance comme modèle pédagogique est très éloquente.

Cette articulation ne peut être véritablement interactive que si, dans un premier temps, l'apprenant identifie dans le stage les difficultés qu'il rencontre (ou que rencontrent les professionnels qu'il observe), les transforme, dans un deuxième temps, en objectifs d'apprentissage, et recherche, dans un troisième 
temps, dans le cadre du dispositif de formation, des ressources lui permettant d'effectuer ces apprentissages et dont la pertinence sera enfin vérifiée, lors des retours en stage. (p. 180)

Ces éléments vont dans le même sens que les différents attributs identifiés par Maubant (2007) pour voir émerger une véritable alternance échappant à l'enfermement organisationnel et permettant d'accompagner le processus d'apprentissage professionnel. Selon cet auteur, un premier attribut, qui consiste à organiser les différents espaces-temps :

selon leur capacité à identifier les savoirs professionnels en jeu dans l'activité d'enseignement et sur leur capacité à accompagner les processus de construction de ces savoirs professionnels [...], s'appuie donc principalement sur un travail préalable de lecture et de compréhension de l'activité d'enseignement, [en faisant] interagir la description compréhensive de cette activité avec les explicitations proposées par l'enseignant. (p. 77)

En ce sens, l'analyse de pratiques, fortement privilégiée dans le document d'orientation de la formation à l'enseignement (Gouvernement du Québec, 2001a), devient centrale et constitue une première modalité pédagogique à mettre en place. Elle rejoint plusieurs écoles de pensée en formation à l'enseignement qui favorisent l'acquisition des compétences professionnelles «par la réflexion sur et dans l'action» (Bourassa, Serre et Ross, 1999; Perrenoud, 1998; Saint-Arnaud, 1992; Schön, 1983). La question de la réflexion sur la pratique est par ailleurs souvent relevée dans les écrits portant sur les stages et la formation pratique à l'enseignement, que cette réflexion soit effectuée à l'écrit ou à l'oral, notamment dans le cadre d'entrevue de rétroaction avec l'enseignant associé ou le superviseur de stage, ou dans le cadre de retour ou séminaire de stage en petits groupes avec le superviseur de stage (Champy-Remoussenard, 2005; Méard et Bruno, 2004; Saussez, Ewen et Girard, 2001). En ce sens, l'accompagnement constitue une deuxième modalité à laquelle plusieurs adhèrent (Arpin et Capra, 2008; Forest et Lamarre, 2009; Hook, McPhail et Vass, 2010; L'Hostie et Boucher, 2004). «Sans l'accompagnement et donc la médiation d'un tiers qui peuvent aider à rendre explicite la pratique professionnelle, l'apprentissage professionnel ne peut fonctionner.» (Maubant, 2007, p. 78)

Par ailleurs, nous avons déjà soulevé la problématique de l'alternance en formation comme praxis pédagogique devant être soutenue par le projet personnel de formation de l'étudiant (Mazalon et Léger 2003). Alors que les recherches sur la formation en alternance proposent quelques définitions de la notion de projet (Bachelard, 1994), Chaix (1993) souligne que l'existence d'un projet professionnel chez l'élève lui permet d'utiliser l'écart entre les différents espaces de formation pour développer des compétences professionnelles en lien avec le métier choisi. Dans un contexte d'alternance en formation, la détermination des projets professionnels «les met en situation de finaliser les contenus de savoir, d'unifier la diversité des voies d'accès au savoir» (p. 205). Sur le plan formatif, la notion de projet devient donc centrale puisque la personne est réellement impliquée dans sa formation. Kaddouri (1994) suggère que la formation ne puisse avoir de sens pour l'individu que si elle est articulée à un projet que celui-ci compte élaborer et qu'elle est cohérente avec les stratégies qu'il met en place pour le réaliser. Ainsi, suivant Geay (1990), pour nous, le modèle d'alternance privilégiée doit permettre à l'étudiant-enseignant «d'avoir une expérience de référence qu'il peut analyser pour donner un sens à ses apprentissages et définir les connaissances à acquérir à l'intérieur de son projet personnel en formation en cohérence avec un projet professionnel» (Mazalon et Léger, 2003, p. 46). 


\subsection{L'alternance intégrative comme choix central pour fonder la formation à l'enseignement professionnel}

Dans un contexte où la majorité des étudiants enseignent, l'équipe du BEP de l'Université de Sherbrooke a voulu développer un projet de formation favorisant l'alternance à la fois comme mode d'organisation et comme stratégie pédagogique de la formation à l'enseignement professionnel. Il faut comprendre que pour une majorité des étudiants, non seulement les stages formels prévus au programme sont utilisés pour l'apprentissage (voir les zones ombragées de la figure 1), mais toute la formation s'appuie sur le partage de leur expérience comme enseignant, dans un va-et-vient incessant entre la pratique d'enseignement dans les établissements de formation professionnelle et la formation les fins de semaine à l'université. En ce sens, le programme de formation élaboré relève d'une alternance dite «intégrative» (Dumont et Wilk, 1992; Mathey-Pierre, 1998), qui vise à une formation théorique et pratique globale, où l'apprenant construit son projet pédagogique, le met en œuvre et effectue un recul réflexif sur cette mise en œuvre (Bachelard, 1994).

La particularité de l'alternance intégrative, souvent considérée comme la véritable alternance, est qu'elle articule une démarche inductive de formation, lorsque les contenus de formation s'appuient sur l'expérience pratique acquise en situation de travail par les apprenants, et une démarche déductive de formation, lorsque la situation de travail est le lieu d'application de la formation (Mathey-Pierre, 1998). L'alternance intégrative inclut également un temps pour l'exploitation pédagogique des expériences spécifiques, voire contradictoires, vécues dans les deux milieux (Fusulier et Maroy, 2002). En somme, l'alternance intégrative part de la personne apprenante avec tous ses acquis scolaires et expérientiels, ses difficultés face au métier d'enseignant, et oblige à questionner les savoirs et les savoir-faire qu'elle mobilise pour ajuster peu à peu expérience et formation aux besoins (Dumont et Wilk, 1992). En somme, parmi les cinq dimensions abordées dans la présentation de l'alternance comme mode d'organisation, et les différents éléments soulevés en ce qui a trait à l'alternance vue comme stratégie pédagogique, l'alternance intégrative commande à notre avis un certain nombre de choix. Parmi ceux-ci, soulignons:

- la prise en compte des trois lieux de formation, soit l'université, les établissements de formation professionnelle et les milieux socioprofessionnels, qui doivent être envisagés à la fois dans leur spécificité et dans leur complémentarité (Fusulier, 2001), comme un seul «espace de formation»;

- la modulation du temps et des rythmes de formation, afin de tenir compte de la réalité des étudiants et des contextes dans lesquels ils évoluent, et ce, en termes de fréquence, durée, périodicité, séquence et localisation temporelle (McGrath et Kelly, 1992, dans Savoie-Zajc, 2001);

- la considération de l'étudiant comme acteur central de sa formation (Chaix, 1993; Geay, 1994; Geay, 1999; Mathey-Pierre, 1998; Mazalon et Bourassa, 2003);

- le caractère essentiel de l'accompagnement de l'étudiant par différents acteurs (enseignant associé, superviseur universitaire, titulaire de cours, par exemple) afin, notamment, de soutenir le passage entre le travail prescrit et le travail réel et de favoriser l'émergence et la construction de sens chez l'alternant (Galvani, 1999), en ayant comme préoccupation constante la cohérence de la formation (Tilman et Delvaux, 2000);

- l'importance accordée à la réflexion sur la pratique et à l'analyse de pratique comme dispositif de formation pour favoriser la construction de savoirs professionnels en enseignement;

- la perspective d'adapter la formation en fonction du projet professionnel de l'étudiant. 
Ces différents éléments constituent les balises théoriques sur lesquelles l'équipe du BEP s'est appuyée pour la conception du programme de formation à l'enseignement professionnel, qui est présenté succinctement dans la prochaine section. Sans entrer dans les détails du programme de baccalauréat en enseignement professionnel de 120 crédits qui a été conçu initialement, nous décrivons brièvement, dans les paragraphes qui suivent, chacune des six phases du programme.

\section{Concevoir le programme de formation en enseignement professionnel de l'Université de Sherbrooke}

Le programme de baccalauréat en enseignement professionnel se présente en six phases (voir figure 1) qui doivent être suivies dans l'ordre prescrit, à l'exception des phases IV et V qui peuvent être faites de façon concomitante. De façon très succincte, nous pouvons dire:

- que la phase I était initialement composée de trois stages réalisés entièrement dans les établissements de formation professionnelle et accompagnés par un enseignant d'expérience appelé mentor;

- que la phase II était initialement constituée de trois blocs cours-stage de sept crédits, alternant les fins de semaines de formation à l'université ( 5 crédits) et la formation en stage dans le milieu scolaire ( 2 crédits);

- que la phase III constituait et constitue toujours une étape bilan permettant à l'étudiant de faire le point sur le développement de ses compétences et de définir ses projets personnels de formation (individuels et collectifs) pour les phases IV et V;

- et que la phase VI est depuis le début constituée d'une seule activité visant à faire un bilan de sa formation et de ses compétences et à définir son projet de développement de carrière en fonction de ce bilan.

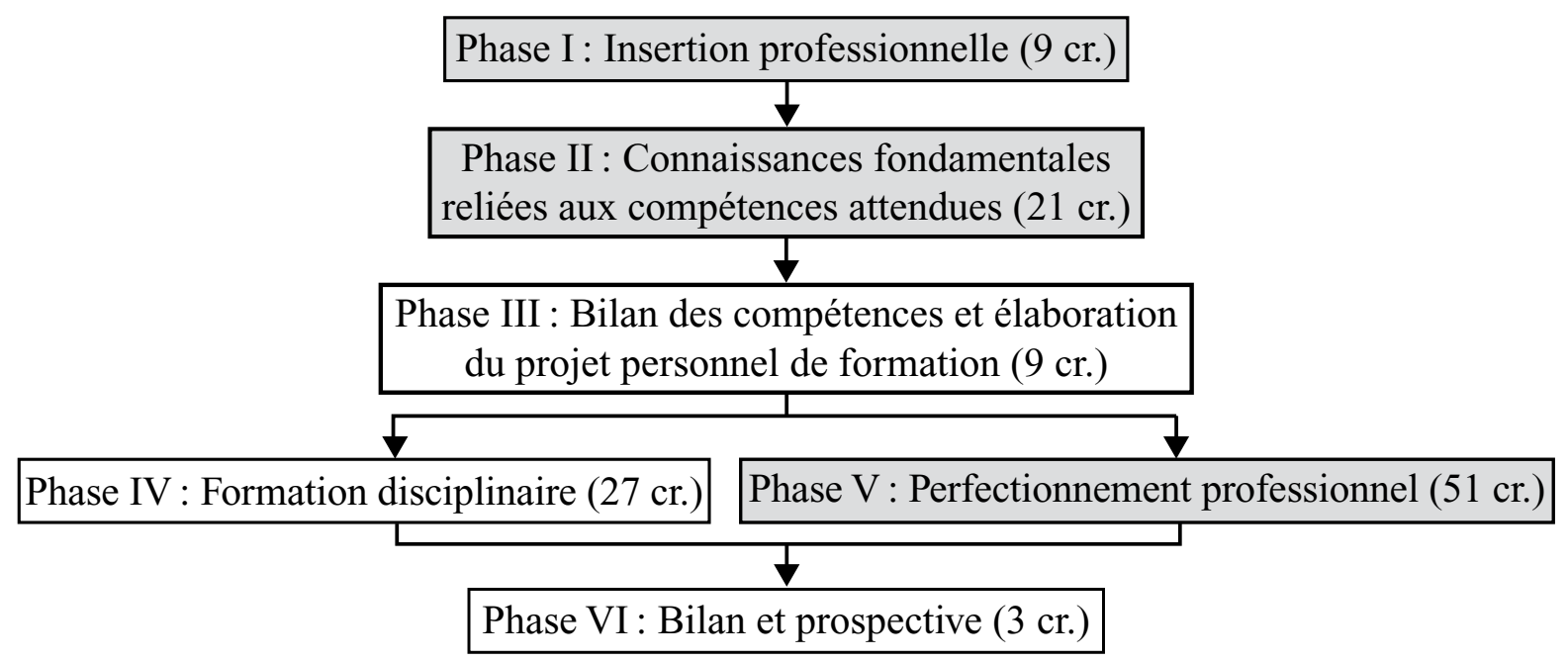

Figure 1 - Les six phases du programme de baccalauréat en enseignement professionnel (BEP) de l'Université de Sherbrooke, tel que conçu initialement 
Puisque relativement peu d'étudiants ont passé à travers tout le processus jusqu'à maintenant et qu'encore peu se trouvent actuellement aux phases III, IV ou V (par conséquent, nous n'avons pas encore recueilli de données à l'égard de ces phases), nous nous limitons, dans les paragraphes qui suivent, à aborder certains éléments de la phase I et de la phase II, en tentant de donner un aperçu des choix qui ont été faits, en traçant un portrait des données qui ont été recueillies et analysées tout au long de la mise en œuvre de ce programme et en indiquant les ajustements qui ont été faits en cours de route, le cas échéant.

\subsection{La phase I: I'alternance d'insertion}

\subsubsection{Description du dispositif de formation}

En raison des caractéristiques et des particularités de l'entrée dans la profession enseignante dans ce secteur, la phase I a été initialement constituée de trois stages de trois crédits qui se déroulent entièrement dans les établissements de formation professionnelle. L'espace de formation est donc partagé entre le milieu scolaire et le milieu professionnel où plusieurs continuent d'exercer le métier. Cette première phase a été conçue dans une grande souplesse en ce qui a trait au temps : l'étudiant peut commencer les stages de la phase I à n'importe quel moment de l'année (localisation temporelle), et effectuer les stages ${ }^{3}$ et les activités propres à chaque stage au rythme (fréquence et périodicité) et dans l'ordre qui lui convient (séquence), de manière à répondre à ses besoins immédiats en termes de planification (IFP 102 - Planification en formation professionnelle), d'intervention (IFP 103 - Intervention en formation professionnelle) et de connaissance du milieu scolaire (IFP 101 - Introduction à la profession enseignante). Ces choix sont effectués en collaboration avec son mentor, un enseignant d'expérience de son milieu scolaire qui l'accompagne dans la réalisation des activités de la phase I. Principalement axée sur la réflexion sur sa pratique, cette phase amène l'étudiant à :

- définir son rôle comme enseignant ainsi que les tâches qui y sont liées, à prendre part aux différentes instances de son milieu scolaire et à se situer par rapport à la profession enseignante (IFP 101);

- prendre conscience de sa pratique en matière de planification et des exigences ministérielles et institutionnelles, adapter sa pratique en fonction de celles-ci, selon une démarche proposée par l'université, et réfléchir sur cette nouvelle façon de faire et l'impact de celle-ci sur son enseignement et sur l'apprentissage de ses élèves (IFP 102);

- prendre conscience de ses façons d'intervenir en classe, élargir son répertoire de possibilités et expérimenter d'autres façons de faire (IFP 103).

Pour accompagner le processus de formation de la phase I, différentes fiches ont été rendues disponibles sur un site internet. Selon la situation, les fiches comprennent des lectures complémentaires, des questions pour amener l'étudiant à décrire sa pratique et à réfléchir sur celle-ci, des capsules vidéo (p. ex.: présentation de différentes formules pédagogiques), etc.

3 Les trois stages sont d'une durée de 135 heures chacun et comportent un certain nombre d'heures de présence en milieu scolaire, et un certain nombre d'heures de travail personnel. 


\subsubsection{Aspects positifs et limites soulevés}

Depuis le début de la mise en œuvre du programme en 2003, différentes rencontres ont eu lieu avec plusieurs centres de formation et commissions scolaires ${ }^{4}$ en vue de faire le point sur la phase I. Sous forme de focus group, ces rencontres regroupaient généralement des étudiants, mentors, conseillers pédagogiques, direction(s) adjointe(s) et direction(s) de centre(s), responsable de la FP à la commission scolaire, etc. De façon plus spécifique, un focus group portant sur l'encadrement et le suivi des stages de la phase I a également été réalisé auprès d'une quinzaine de mentors expérimentés ${ }^{5}$ de diverses commissions scolaires de la région montréalaise (Gagnon, Beaucher, Rousseau et Lehouillier, 2007). Enfin à la fin de l'année 2006, un questionnaire a été envoyé à l'ensemble des mentors œuvrant dans le programme ${ }^{6}$ et portait sur les conditions d'accompagnement des stagiaires en enseignement professionnel en phase I (Rousseau, 2008). L'analyse des nombreuses données recueillies a permis de constater les éléments positifs et a soulevé quelques difficultés et limites de ce dispositif.

D'une part, en termes de contenu, les étudiants apprécient de façon générale les activités de stage qui sont directement liées à leur travail et leur permettent de développer leurs compétences en enseignement, mais relèvent un certain nombre d'éléments «inutiles et trop complexes» à cette étape de leur carrière; ils apprécient aussi pouvoir commencer leur formation à n'importe quel moment de l'année, et pouvoir effectuer cette formation à leur rythme en étant accompagnés par un enseignant d'expérience dans leur milieu. Dans le même sens, les mentors, les conseillers pédagogiques, les directions de centres de formation et les directions de commissions scolaires sont tous d'avis que la phase I permet au nouvel enseignant d'acquérir rapidement les compétences de base en enseignement, ce qui facilite grandement leur insertion dans la profession et permet de pouvoir compter à court terme sur des ressources qualifiées. Les étudiants ont manifesté le besoin de rencontrer les représentants de l'Université avant la fin de la phase I; ils se sentent isolés, ils ont l'impression de n'avoir pas toujours une vision claire du parcours à venir au programme et de demeurer incertains face à leurs capacités à être étudiant «dans» l'université. En ce qui a trait aux ressources, les mentors et les étudiants soulignent la qualité du matériel qui leur est fourni, et les mentors considèrent les guides d'accompagnement comme des outils indispensables à l'exercice de leur rôle. Ces derniers indiquent également que la formation offerte leur permet d'approfondir le contenu de chacun des stages et de se familiariser avec le matériel d'accompagnement afin d'obtenir une idée précise et concrète des tâches des stagiaires et du rôle qui leur incombe comme accompagnateur. Comme les étudiants, les mentors disent cependant se sentir isolés et laissés à eux-mêmes dans leur rôle; ils aimeraient des contacts plus fréquents entre l'université et les mentors (suivis téléphoniques et rencontres), notamment pour discuter du cheminement des stagiaires, et sont en faveur d'une première activité qui se déroulerait à l'université pour les étudiants. Ils soulèvent que

4 Depuis 2005, une dizaine de centres ou commissions scolaires ont été rencontrés, dans différentes régions desservies par le programme, notamment Saint-Hyacinthe, Laval, Victoriaville, Laurentides, Montréal, Montérégie, Sherbrooke, Québec.

5 Précisons que ces personnes sont enseignantes ou conseillères pédagogiques. À l'exception d'une seule, elles étaient mentors depuis au moins 3 ans, et pour chacune, le nombre de stagiaires accompagnés à titre de mentor varie de 2 à plus de 15. Cinq d'entre elles étaient aussi enseignantes associées (phase II).

6447 sujets ayant accompagné des stagiaires du BEP de l'Université de Sherbrooke depuis août 2004 (régions du Bas-Saint-Laurent, de la Capitale-Nationale, du Centre-du-Québec, de la Chaudière-Appalaches, de l'Estrie, de Lanaudière, des Laurentides, de Laval, de la Mauricie, de la Montérégie, de Montréal et de l'Outaouais). De ce nombre, 153 personnes ont répondu (taux de réponse de 34,2\%). 
la grande flexibilité du rythme de formation se traduit parfois en démotivation et laxisme en regard de la durée des stages et sont en faveur de l'imposition d'un temps limite pour la réalisation des stages de la phase I, tout en permettant une certaine flexibilité pour les cas particuliers. Enfin, la lourdeur de la tâche d'accompagnement dans ce contexte et le manque de reconnaissance de leur rôle dans leur milieu et par l'université sont par ailleurs fortement décriés.

\subsubsection{Modifications apportées}

Pour répondre aux besoins des étudiants, des intervenants et des différentes personnes concernées par la formation des enseignants en formation professionnelle, plusieurs éléments ont été modifiés, enlevés ou ajoutés dans le processus de formation de la phase I. Sans vouloir entrer dans le détail de chacune des modifications, nous nous contenterons ici de nommer les principales:

- révision complète des trois stages en vue de limiter le contenu ${ }^{7}$ à ce qui est essentiel à cette étape du développement des compétences des étudiants et de leur carrière en enseignement;

- création d'une activité d'accueil et d'intégration (IFP 100) à l'université avant le début des stages, dans un but de familiarisation des étudiants avec le monde universitaire, dans un contexte agréable, dynamique et rassurant, cette activité aborde certains contenus qui ont été retranchés des stages, notamment du IFP 101;

- ajout de superviseurs en phase I, qui sont appelés à faire des suivis téléphoniques et des rencontres avec les étudiants et les mentors, à prendre la responsabilité de l'évaluation, à effectuer des vérifications dans les cas qui semblent problématiques, etc.

\subsection{La phase II : I'alternance de qualification}

\subsubsection{Description du dispositif de formation}

Élaborée dans une perspective de professionnalisation des enseignants de la formation professionnelle, cette phase était initialement composée de trois blocs de sept crédits ${ }^{8}$, chacun des blocs pouvant être réalisé à l'intérieur d'une session universitaire (durée), et ce, dans n'importe quel ordre (séquence), et visant l'acquisition des connaissances fondamentales reliées aux compétences en enseignement et leur mise en œuvre dans la pratique professionnelle de l'enseignant stagiaire: «Conception et planification des situations d'apprentissage», «Pilotage et mise en œuvre des situations d'apprentissage» (intervention), et «Évaluation des apprentissages et des enseignements ».

Ainsi, parce qu'elle alterne de façon régulière (fréquence et périodicité) entre les deux espaces de formation que sont la formation à l'université et les stages dans les établissements de formation professionnelle ${ }^{9}$, l'alternance de la phase II a été conçue comme un va-et-vient visant à faciliter

7 Cette révision du contenu a également mené à la conception de nouveaux documents de formation qui ont été déposés sur une nouvelle plateforme internet plus conviviale, et à la conception de nouveau matériel d'accompagnement pour les mentors.

8 Ces sept crédits correspondent à cinq crédits pour les cours et deux crédits pour les stages.

9 Bien que le milieu de travail (lié au métier initial) soit encore présent pour plusieurs étudiants, l'accent est mis, au cours de cette phase, sur l'alternance entre l'université et l'établissement de formation professionnelle. 
l'intégration des savoirs théoriques et des savoirs expérientiels, en permettant aux étudiants de donner du sens à leur formation (Gagnon et Mazalon, 2008). Structurée de façon à respecter le plus fidèlement possible les principes de l'alternance intégrative (Dumont et Wilk, 1992; Mathey-Pierre, 1998), la formation inclut, dans les périodes de stages, à la fois des activités de nature déductive, qui visent l'application des connaissances acquises lors de la fin de semaine de cours précédant le stage, et des activités de nature inductive, sur lesquelles s'appuiera l'acquisition des connaissances enseignées lors de la fin de semaine de cours suivante. En plus des apports théoriques, les fins de semaine de formation comprennent un temps spécifique pour effectuer un retour sur le stage et un temps dédié au départ ou à la préparation pour le stage. Pour la réalisation de cette phase, l'étudiant dispose de trois documents d'accompagnement (guide de stage, guide de formation, manuel de référence) en plus de divers outils fournis en classe et de documents du Ministère sur lesquels il doit appuyer sa pratique (programme d'étude, guide d'évaluation, etc.). En termes de relations, trois personnes interviennent dans le processus de formation de la phase II : le titulaire du cours en charge des fins de semaine de formation, l'enseignant associé dans le milieu scolaire ${ }^{10}$, et le superviseur universitaire, qui anime les retours de stage les samedis matins, effectue différentes activités dans le milieu scolaire (observations et autres), accompagne un groupe d'étudiants et leurs enseignants associés dans la réalisation des activités de stage et fait le lien entre les différents acteurs et les espaces de la formation.

\subsubsection{Aspects positifs et limites soulevés}

Outre les différentes évaluations ${ }^{11}$ effectuées à chaque session depuis la première mise en œuvre d'un bloc cours-stage à l'hiver 2005, un sondage à l'hiver $2008^{12}$ a été réalisé auprès des étudiants à propos d'éléments relatifs au temps, et un questionnaire d'opinions a été distribué à l'automne $2009^{13}$ en vue de connaitre l'appréciation des étudiants à l'égard du processus de formation de la phase II et leur intérêt face à une formule davantage médiatisée ${ }^{14}$. Depuis le début de la mise en œuvre, différents focus group ont également eu lieu avec des superviseurs, des enseignants associés et des titulaires, et ces derniers ont également répondu à différentes évaluations à chaque session concernant l'appréciation du processus de formation et des relations entre les intervenants. Nous relevons ici quelques éléments qui ont mené à des modifications du processus de formation.

En ce qui a trait à la dimension temps, plusieurs questionnent l'ordonnancement des trois blocs en indiquant que le bloc sur la planification devrait obligatoirement se faire en premier. La

10 Comme le mentor en phase I, il s'agit d'un enseignant d'expérience qui accompagne l'étudiant dans la réalisation des activités de stage.

11 À chaque session, chaque étudiant inscrit à un bloc cours-stage de la phase II complète les évaluations suivantes: Appréciation du stagiaire au regard des activités de stage, Appréciation du stagiaire au regard de l'accompagnement reçu par la personne enseignante associée, Appréciation du stagiaire au regard de l'accompagnement reçu par la personne superviseure universitaire et Questionnaire d'évaluation générale du cours (lié à l'enseignement des fins de semaine de formation).

12 Ce sondage a été effectué auprès de tous les étudiants inscrits en phase II à ce moment (154 réponses sur 160), soit 27 étudiants à Gatineau, 56 à Longueuil, 23 à Québec, 19 à Sherbrooke et 29 à Trois-Rivières.

13 Ce questionnaire a été distribué à tous les étudiants inscrits en phase II à ce moment (199 réponses sur 226), soit 1 étudiant à Gatineau, 34 à Laval, 94 à Longueuil, 25 à Québec, 32 à Sherbrooke et 29 à Trois-Rivières.

14 Cet élément a fait l'objet d'une partie du questionnaire après la demande d'un certain nombre d'étudiants d'avoir des cours en ligne plutôt qu'en personne les fins de semaine. 
majorité s'entend pour dire que les activités proposées dans le cadre des stages prennent trop de temps et voit celles-ci comme un ajout substantiel à leur travail. Plusieurs soulignent la difficulté de réaliser sept crédits universitaires à l'intérieur d'une session compte tenu du fait qu'ils travaillent à temps plein et qu'ils ont des familles, et souhaitent avoir le choix de le faire sur deux sessions. Certains trouvent également difficile de devoir effectuer certaines activités de façon spécifique au cours d'une séquence de stage (localisation temporelle), compte tenu de leur tâche et horaire d'enseignement variable ${ }^{15}$ et de l'endroit où ils sont rendus dans l'enseignement de leur module à un moment particulier. De même, en termes de contenu, certains contextes d'enseignement se prêtent mal à la réalisation de plusieurs activités ${ }^{16}$. Aussi, sur le plan pédagogique, une majorité d'étudiants critique le processus d'analyse réflexive. Bien que certains étudiants l'apprécient, ils montrent une capacité d'analyse exemplaire de leurs pratiques et en voient la pertinence, plusieurs titulaires considèrent par ailleurs que ce processus est bâclé par beaucoup d'étudiants et ne permet pas d'atteindre les objectifs visés. Les titulaires soulignent aussi l'hétérogénéité de la qualité de la formation qui semble avoir été faite au cours de la phase $\mathrm{I}^{17}$ et la nécessité d'effectuer une certaine mise à niveau au début du processus de formation de la phase II. Par ailleurs, en ce qui concerne les différents intervenants, l'arrimage demeure difficile et renvoie parfois des messages contradictoires aux étudiants (Gagnon et Mazalon, 2008). De même en ce qui a trait aux outils de formation, les étudiants, bien qu'ils reconnaissent la qualité de ceux-ci, expriment la difficulté de se retrouver devant le nombre important de documents, et souhaiteraient que le manuel de référence, le guide des activités de formation et le guide des activités de stage soient regroupés à l'intérieur d'un seul et même document.

\subsubsection{Modifications apportées ou à venir}

À la suite de ces différents constats, plusieurs modifications ont été apportées à chaque session. Entre autres, mentionnons l'adaptation de plusieurs activités de stage à différents contextes d'enseignement (enseignement individualisé, enseignement dans le cadre de stage dans le secteur de la santé, etc.); l'identification de différentes activités «mobiles» pouvant être réalisées au moment le plus opportun pour l'étudiant; la souplesse accordée à certaines activités devant être obligatoirement réalisées à l'intérieur d'une séquence spécifique de stage; la diminution du nombre et de l'ampleur des activités à réaliser à chaque séjour de stage; la modification de l'offre de cours, permettant aux étudiants de réaliser un bloc cours-stage sur une ou deux sessions; 1'ajout d'une activité d'un crédit avant le début de la phase II (bilan et transition) permettant d'effectuer un bilan des apprentissages, d'effectuer une certaine mise à niveau; le retrait ou la modification de certains éléments des manuels de référence; etc.

Par ailleurs, tant d'un point de vue organisationnel que pédagogique, la phase II est actuellement en révision majeure, une révision fondée sur ce qui se fait dans d'autres secteurs de formation, notamment en ce qui a trait à l'analyse réflexive (Saintonge et Gallagher, 2010).

15 Par exemple, il peut être difficile pour une étudiante qui enseigne dans le secteur de la santé d'enseigner une leçon en expérimentant telle stratégie d'enseignement alors que pendant la période où il lui est demandé de le faire, elle a uniquement une tâche de supervision de stagiaires en milieu hospitalier.

16 Par exemple, plusieurs programmes de formation professionnelle sont offerts en enseignement individualisé alors que bon nombre d'activités de stage du bloc cours-stage portant sur la planification amènent les étudiants à planifier et à mettre en œuvre des leçons selon différents critères.

17 Certaines modifications apportées en phase I visaient également à répondre à cette problématique. 


\section{Confronter l'alternance à la réalité : quelques éléments de discussion en guise de conclusion}

Considérée non seulement comme mode d'organisation de la formation mais également comme stratégie pédagogique, l'alternance du BEP de l'Université de Sherbrooke est fortement ancrée dans le contexte réel de la pratique et la conception du programme a tenté de respecter le plus possible le principe pédagogique du mouvement itératif entre les milieux de formation, en vue de favoriser l'articulation "théorie-pratique» et de permettre le développement de compétences tant recherchées dans la formation à un métier tel que l'enseignement (Mazalon et Landry, 1998; Mazalon et Léger, 2003). Ainsi, l'alternance du BEP se voulait intégrative (Dumont et Wilk, 1992) en permettant « d'articuler ensemble, dans le même temps et non dans des processus de formation séparés, la formation aux savoirs et la formation aux savoir-faire dans le contexte réel de l'action » (Gouvernement du Québec, 2001a, p. 32), et en passant notamment par l'analyse réflexive sur les pratiques et le projet personnel de formation, deux composantes centrées sur l'individu mais dont l'accompagnement constitue un élément essentiel.

La mise en œuvre et l'évaluation des deux premières phases du BEP a cependant relevé certaines limites à l'alternance dans la formation des enseignants en formation professionnelle. Ces limites ont trait, notamment, aux particularités des enseignants/étudiants de ce secteur et aux différents contextes dans lesquels ils sont appelés à enseigner. Lier les différents contenus d'enseignement aux activités réalisées dans le cadre du travail en enseignement ne s'avère pas toujours facile. Effectuer certaines activités pour développer les compétences telles qu'elles sont prescrites par le MELS relève dans certains contextes d'enseignement de la magie. Développer l'analyse réflexive, du moins à l'écrit, se heurte à la difficulté et au manque d'intérêt, pour ces personnes «axées sur l'action» et dont la compétence liée à la langue écrite est souvent limitée, de réfléchir sur leur pratique de façon formelle à l'intérieur d'un travail universitaire. S'assurer d'une compréhension univoque des rôles et responsabilités de chacun des intervenants demeure complexe ${ }^{18}$ lorsqu'on souhaite établir une saine collaboration entre chacune des parties et, en définitive, favoriser la réussite des étudiants dans le BEP. Accompagner les étudiants de façon adéquate et professionnelle dans chacun des espaces de formation s'avère tout un défi, alors qu'il est parfois difficile, dans certains milieux, de recruter et de maintenir dans leur rôle des enseignants intéressés à accompagner des stagiaires en formation professionnelle, que les enseignants d'expérience désignés dans les centres de formation professionnelle ne sont pas passés par ce processus de formation, et qu'ils sont peu reconnus et peu soutenus dans leur rôle d'accompagnateur (Rousseau, 2008). À cet égard, il importe à notre avis de faire des représentations auprès des dirigeants des établissements de formation professionnelle et des commissions scolaires afin de les sensibiliser à l'importance de valoriser et de reconnaitre le rôle des personnes qui accompagnent les stagiaires en enseignement professionnel.

Enfin, dans un contexte où la tradition d'encadrement de stagiaires est encore jeune, où tout est à définir et à mettre en œuvre (Comité d'orientation de la formation du personnel enseignant, 2005), l'organisation de la formation pratique en formation professionnelle dans un contexte d'alternance intégrative a nécessité et nécessite encore des efforts considérables tant de la part du réseau

18 Dans un contexte où il y a quelques centaines d'intervenants qui œuvrent en même temps dans les différentes régions du Québec, cela soulève des enjeux importants au plan de la gestion pédagogique des ressources humaines lorsque l'on souhaite organiser une formation uniforme et de qualité sur l'ensemble du territoire. 
universitaire que de celui du réseau scolaire. Aussi, avons-nous le défi de poursuivre le développement de relations de partenariat avec les milieux scolaires et de consolider ces relations en vue de trouver des solutions permettant de développer des programmes de formation à l'enseignement professionnel favorisant les aspects pédagogiques et didactiques de l'apprentissage professionnel (Maubant, 2007) dans un contexte d'ingénierie de l'alternance en formation universitaire.

\section{Références}

Aballea, F. (1991). Alternance, qualifications et insertion professionnelle. Recherche sociale, 118(avril-juin), 3-43.

Agulhon, C. (2000). L'alternance: une notion polymorphe, des enjeux et des pratiques segmentées. Revue française de pédagogie, 131(avril-mai-juin), 55-63.

Arpin, L. et Capra, L. (2008). Accompagner l'enseignant dans son parcours professionnel. Montréal: Chenelière Éducation.

Audet, C. (1995). L'alternance en formation professionnelle au secondaire: défis, limites et conditions de réalisation. Québec: Conseil supérieur de l'éducation, Service des études et de la recherche.

Bachelard, P. (1994). Apprentissage et pratiques d'alternance. Paris : L'Harmattan.

Baillauquès, S. , Lavoie, M., Chaix, M.-L. et Hétu, J.-C. (2002). L’identité chez les formateurs d'enseignants. Échanges franco-québécois. Paris: L'Harmattan.

Balleux, A. (2003). La formation professionnelle: une formation pratique marquée par le passage du métier à celui de l'enseignement, In A. Balleux et M. Tardif (dir.), La formation à l'enseignement professionnel: identité, enjeux et perspectives. Actes du $20^{\mathrm{e}}$ Congrès de l'AIPU (p. 18-28). Sherbrooke: Éditions du CRP.

Balleux, A. (2006a). Les étudiants en formation à l'enseignement professionnel au Québec: portrait d'un groupe particulier d'étudiants universitaires. Revue canadienne d'enseignement supérieur, 36(1), 29-48.

Balleux, A. (2006b). L'entrée en enseignement professionnel au Québec : 1'apport du processus migratoire à la lecture d'un mouvement de passage. Carriérologie, 10(3-4), 603-627.

Balleux, A. et Loignon, K. (2004). Quitter le métier pour l'enseignement. Rapport de recherche sur l'identité des enseignants débutants en formation professionnelle au Québec. Document non publié. Sherbrooke: Université de Sherbrooke, Faculté d'éducation.

Beaucher, C., Chatigny, C., Balleux, A., Tardif, M. et Gagnon, C. (2005). Nouveaux étudiants, nouvelles exigences, nouveaux besoins: l'adaptation et la mise en œuvre d'un programme innovant de formation des maitres à l'enseignement professionnel au Québec. Communication présentée dans le cadre du congrès de l'AIPU, Genève, 12-14 septembre.

Beaucher, C. et Gagnon, C. (2008). Accueillir, guider, accompagner : des mesures innovantes pour assurer la persévérance des étudiants du baccalauréat en enseignement professionnel de l'Université de Sherbrooke. Communication présentée dans le cadre du congrès de l'AIPU, Montpellier, 19 au 22 mai.

Boënnec, M. et Maurel-Indart, H. (2001). Guide de l'étudiant stagiaire en entreprise: de la recherche du stage à la soutenance du rapport. Paris: Vuibert.

Bourassa, B., Serre, F. et Ross, D. (1999). Apprendre de son expérience. Québec: Presses de l'Université du Québec.

Bourgeon, G. (1979). Socio-pédagogie de l'alternance. Paris : UNMFREO.

Boutet, M. et Pharand, J. (dir.) (2008). L'accompagnement concerté des stagiaires en enseignement. Québec: Presses de l'Université du Québec.

Boutet, M. et Rousseau, N. (2002). Les enjeux de la supervision pédagogique des stages. Québec: Presses de 1'Université du Québec.

Boutin, G. (2002). Formation pratique des enseignants et partenariat. État des lieux et prospective. Montréal : Éditions Nouvelles.

Candau, G. et Kittel, F. (2001). Situation pédagogique de l'alternance et construction du savoir de l'apprenant. In S. Perez et O. Strobel (dir.), Éducation et travail: divorce ou entente cordiale? (p. 97-112). Paris: L'Harmattan.

Caron, L. (1997). Rapport de recherche sur la condition enseignante en formation professionnelle. Synthèse. Québec: FECS, Centrale des enseignants du Québec. 
Chaix, M.-L. (1993). Se former en alternance. Le cas de l'enseignement technique agricole. Paris: L'Harmattan.

Champy-Remoussenard, P. (2005). Écritures sur l'activité professionnelle et pédagogie de l'alternance dans l'enseignement supérieur. In C. Hahn, M. Besson, B. Colin et A. Geay (dir.), L'alternance dans l'enseignement supérieur. Enjeux et perspectives (p. 319-336). Paris: L'Harmattan.

Charland, J.-P. (1982). Histoire de l'enseignement technique et professionnel. L'enseignement spécialisé au Québec, 1867 à 1982. Québec: Institut québécois de recherche sur la culture.

Clénet, J. (2002). L'ingénierie des formations en alternance. Pour comprendre, c'est-à-dire pour faire... Paris: L'Harmattan.

Conseil supérieur de l'éducation (1984). Vers des aménagements de la formation et du perfectionnement des enseignants du primaire et du secondaire: commentaires sur un projet ministériel: avis au ministre de l'éducation. Québec: Direction des communications du Conseil supérieur de l'éducation.

Conseil supérieur de l'éducation (1992). En formation professionnelle: l'heure d'un développement intégré. Avis au ministre de l'Éducation et à la ministre de l'Enseignement supérieur et de la Science. Québec: Direction des communications du Conseil supérieur de l'éducation.

Comité d'orientation de la formation du personnel enseignant (1998). Une formation de qualité pour un enseignement professionnel. Avis à la Ministre. Québec: Ministère de l'Éducation.

Comité d'orientation de la formation du personnel enseignant (2005). La formation en milieu de pratique: de nouveaux horizons à explorer. Avis au Ministre. Québec: Ministère de l'Éducation, du Loisir et du Sport.

Correa Molina, E. et Gervais, C. (2008). Les stages en formation à l'enseignement. Pratiques et perspectives théoriques. Québec: Presses de l'Université du Québec.

Correa Molina, E, Gervais, C. et Rittershaussen, S. (2008). Vers une conceptualisation de la situation de stage: explorations internationales. Sherbrooke: Éditions du CRP.

Danvers, F. (1992). 700 mots-clefs pour l'éducation. Québec: Les Presses de l'Université Laval.

De Cock, G. (2001). Application d'un modèle de réflexion sur la pratique à des journaux de bord utilisés lors de stages. Mémoire de Licence en psychologie et en sciences de l'éducation, Université catholique de Louvain.

Dumont, M. et Wilk, D. (1992). Pour une alternance qualifiante. Paris: Centre INFFO.

Engeström, Y., Engeström, R. et Kärkkäinen, M. (1995). Polycontextuality and boundary crossing in expert cognition: Learning and problem solving in complex work activities. Learning and Instruction, 5, 319-336.

Forest, L. et Lamarre, A.M. (2009). Accompagner des stagiaires vers la maitrise des compétences en enseignement. Montréal: Chenelière Éducation.

Fusulier, B. (2001). Articuler l'école et l'entreprise. Paris: L'Harmattan.

Fusulier, B., et Maroy, C. (2002). La formation en alternance en Belgique francophone: développements pratiques et théoriques. In C. Landry (dir.), La formation en alternance: état des pratiques et des recherches (p. 109-134). Québec: Presses de l'Université du Québec.

Gagnon, C. (2007). Arrimage des pratiques éducatives d'enseignants et de formateurs en entreprises en contexte d'alternance. Études de cas en formation professionnelle agricole. Thèse de doctorat inédite en éducation, Université de Sherbrooke.

Gagnon, C. (2008). Arrimage des pratiques éducatives d'enseignants et de formateurs en entreprises en contexte d'alternance. Études de cas en formation professionnelle agricole. Recherches qualitatives, 27(1), 141-190.

Gagnon, C., Beaucher, C, Rousseau, A. et Lehouillier, L. (2007). Implantation d'un programme en enseignement professionnel: suivi de la mise en æuvre des stages en début de formation. Communication présentée dans le cadre du congrès de l'AIPU, Montréal, 16 au 18 mai.

Gagnon, C. et Mazalon, É. (2008). L'approche qualité dans la formation à l'enseignement professionnel en alternance: la nécessaire collaboration des acteurs. Communication présentée dans le cadre du congrès de l'AIPU, Montpellier, 19 au 22 mai.

Gagnon, C. et Mazalon, É. (2010a). Alternances et professionnalisation: regards complexes sur la formation des maitres en enseignement professionnel au Québec. Communication présentée au symposium international «Formations des adultes, alternances et professionnalisations : des recherches à l'épreuve de la complexité», dans le cadre du colloque international «La pensée complexe: défis et opportunités pour l'éducation, la recherche et les organisations», Université de Lille 1, France, 31 mars et $1^{\text {er }}$ avril. 
Gagnon, C. et Mazalon, É. (2010b). L'alternance en formation à l'enseignement professionnel: partenariat, accompagnement et professionnalisation. Communication présentée dans le cadre du congrès annuel de la Société canadienne pour l'étude de l'éducation (SCÉÉ), Université Concordia, Montréal, 29 mai au $1^{\text {er juin. }}$

Galvani, P. (1999). Fertilisation croisée des savoirs et ingénierie d'alternance socio-formative. Le programme de recherche-formation-action Quart Monde/Université. Revue française de pédagogie, 128(juillet-août-septembre), 25-34.

Geay, A. (1990). Stratégie d'apprentissage et stratégie d'enseignement en système de formation alternée. Éducation permanente, 106, 51-58.

Geay, A. (1994). Une alternative au «Tout école». Cahiers pédagogiques, 320(janvier), 16-17.

Geay, A. (1999). Actualité de l'alternance. Revue française de pédagogie, 128(juillet-août-septembre), 107-125.

Geay, A. et Sallaberry, J.-C. (1999). La didactique en alternance ou comment enseigner dans l'alternance? Revue française de pédagogie, 128(juillet-août-septembre), 7-15.

Gérard, C. (2000). Pragmatique de l'alternance et approche systémique. Construire du sens en problématisant. Les dossiers des sciences de l'éducation, numéro spécial sur les approches systémiques et recherches en sciences de l'éducation, 3, 27-40.

Gervais, F. (1995). Superviseurs universitaires et formation pratique en milieu scolaire: orientation de la médiation entre théorie et pratique. Revue des sciences de l'éducation. XXI(3), 541-561.

Gervais, C. et Desrosiers, P. (2005). L'école, lieu de formation d'enseignants. Questions et repères pour l'accompagnement de stagiaires. Québec: Les Presses de l'Université Laval.

Gimonet, J.-C. (1998). Alternance et formation. Associer la théorie à la pratique. L'information agricole, 707, $27-28$.

Gomez, F. et Hostein, B. (1997). Mémoire de formation professionnelle et alternance. In D. Violet (dir), Formation d'enseignants et alternances (p. 39-73). Paris: L'Harmattan.

Gouvernement du Québec (1999). Prévisions de l'effectifenseignant des commissions scolaires de 1996-1997 à 20082009. Bulletin statistique de l'éducation, n 9. Québec: Ministère de l'Éducation.

Gouvernement du Québec (2001a). La formation à l'enseignement professionnel. Les orientations. Les compétences professionnelles. Québec: Ministère de l'Éducation.

Gouvernement du Québec (2001b). L'alternance en formation professionnelle et technique, cadre de référence. Québec: Ministère de l'Éducation du Québec, Secteur de la formation professionnelle et technique.

Groupe de travail sur la relance de la formation professionnelle des jeunes au secondaire et de la formation technique (1995). La formation professionnelle chez les jeunes: un défi à relever. Québec: Ministère de l'Éducation, Direction des communications.

Hardy, M., Desrosiers-Sabbath, R. et Defrênes, E. (1995). Modalités de socialisation et représentation didactique de maitres de l'enseignement professionnel au Québec. Revue des sciences de l'éducation, XXI(4), 809-830.

Hook, P., McPhail, I. et Vass, A. (2010). L'accompagnement en éducation et la pratique réflexive (Trad. par M. St-Germain). Montréal: Chenelière Éducation.

Kaddouri, M. (1994). Paque: l'alternance et ses modalités. Actualité de la formation permanente, 130(mai-juin), 89-98.

Landry, C. (1992). Les relations interorganisationnelles dans la formation en alternance pour des jeunes adultes. Thèse de doctorat en éducation, Université de Montréal.

Lerbet-Séréni, F. et Violet, D. (1999). Paradoxes de la médiation. Tradition et alternance. Revue française de pédagogie, 128(juillet-août-septembre), 17-24.

L'Hostie, M. et Boucher, L.-P. (dir.) (2004). L'accompagnement en éducation: un soutien au renouvellement des pratiques. Québec: Presses de l'Université du Québec.

Malglaive, G., et Weber, A. (1983). École et entreprise: intérêt et limites de l'alternance en pédagogie. Revue française de pédagogie, 62(janv.-fév.-mars), 51-64.

Martin, D., Garant, C., Gervais, C. et St-Jarre, C. (2000). Recherches et pratiques en formation des maitres. Vers une pratique réfléchie et argumentée. Sherbrooke: Éditions du CRP.

Mathey-Pierre, C. (1998). Alternance (Formations en). In P. Champy et C. Étévé (dir.), Dictionnaire encyclopédique de l'éducation et de la formation (p. 71-73). Paris: Nathan. 
Maubant, P. (2007). Penser l'alternance comme logique de professionnalisation des enseignants. In F. Merhan, C. Ronveaux et S. Vanhulle (dir.), Alternances en formation (p. 67-82). Bruxelles: De Boeck.

Mazalon, É., Beaucher, C. et Langlois, M. (2010). L'évolution et la détermination des projets d'élèves inscrits dans un programme de formation professionnelle en alternance travail-études. Revue de recherche appliquée sur l'apprentissage, 3, 1-20.

Mazalon, É. et Bourassa, B. (2003). L'alternance en formation professionnelle au Québec: du dispositif institutionnel à la pratique des acteurs. In M. Hardy (dir.), Concertation éducation-travail: politiques et expériences (p. 187209). Québec: Presses de l'Université du Québec.

Mazalon, É. et Landry, C. (1998). L'alternance au Québec, une idée ancienne pour de nouvelles pratiques de formation. Cahiers de la recherche en éducation, 5(1), 93-116.

Mazalon, É. et Léger, C. (2003). L'alternance en formation des maitres: une praxis pédagogique soutenue par le projet personnel en formation. In G. Boutin (dir.), La formation des enseignants en question. Modalités, entrée dans le métier et dimensions critiques (p. 35-53). Montréal: Éditions Nouvelles.

McGrath, J.E. et Kelly, J.R. (1992). Temporal context and temporal patterning: Toward a time-centered perspective for social psychology. Time and society, 1(3), 399-420.

Méard, J. et Bruno, F. (2004). L'analyse de pratique au quotidien. 32 outils pour former les enseignants. Nice: CRDP de l'Académie de Nice.

Meirieu, P. (1992). Apprendre... oui, mais comment. Paris: ESF.

Merhan, F. et Baudouin, J.-M. ( 2007). Alternance, exotopie et dynamiques identitaires. Enjeux et significations du rapport de stage. In F. Merhan, C. Ronveaux et S. Vanhulle (dir.), Alternances en formation (p. 203-223). Bruxelles: De Boeck.

Morandi, F. (1999). Alternance cognitive et alternance formative dans la formation professionnelle des professeurs des écoles: représentations et «actions connaissantes». Revue française de pédagogie, 128(juillet-août-septembre), 43-54.

Perrenoud, P. (1998). De l'alternance à l'articulation entre théories et pratiques dans la formation initiale des enseignants. In M. Tardif, C. Lessard et C. Gauthier (dir.), Formation des maitres et contextes sociaux. Perspectives internationales (p. 153-199). Paris: Presses universitaires de France.

Portelance, L. et Tremblay, F. (2006). Les responsabilités complémentaires de l'enseignant associé et du stagiaire au regard de la formation à l'enseignement. In J. Loiselle, L. Lafortune et N. Rousseau (dir.), L'innovation en formation à l'enseignement. Pistes de réflexion et d'action (p. 43-54). Québec: Presses de l'Université du Québec.

Reviol, A. (2001). Enseigner en Suisse. Un rapport au temps empreint de tensions spécifiques. In C. St-Jarre et L. Dupuy-Walker (dir.), Le temps en éducation: regards multiples (p. 163-192). Québec: Presses de l'Université du Québec.

Rousseau, A. (2008). Conditions visant à favoriser et à soutenir la motivation des enseignantes et enseignants $\grave{a}$ accompagner des stagiaires en enseignement professionnel. Essai de maitrise en gestion de l'éducation et de la formation, Université de Sherbrooke.

Saint-Arnaud, Y. (1992). Connaitre par l'action. Montréal: Les Presses de l’Université de Montréal.

Saintonge, L. et Gallagher, F. (2010). La pertinence d'une formation pour encourager la pratique réflexive en contexte de stage et en contexte réel de travail. Communication présentée dans le cadre du Mois de la pédagogie universitaire, Université de Sherbrooke, 21 avril.

Saussez, F., Ewen, N. et Girard, J. (2001). Au cœur de la pratique réflexive, la conceptualisation? Réflexions à partir d'un dispositif de formation au Grand-Duché du Luxembourg. Recherche et formation, 36, 69-87.

Savoie-Zajc, L. (2001). Les facteurs temporels dans l'alternance étude-travail en formation professionnelle au secondaire. In C. St-Jarre et L. Dupuy-Walker (dir.), Le temps en éducation: regards multiples (p. 351-371). Québec: Presses de l'Université du Québec.

Schneider, J. (1999). Réussir la formation en alternance. Organiser le partenariat jeune-entreprise-organisme de formation. Paris: INSEP.

Schön, D.A. (1983). The reflective practitioner: How professionals think in action. New York, NY: Basic Books.

Tardif, M. et Lessard, C. (1999). Le travail enseignant au quotidien. Contribution à l'étude du travail dans les métiers et les professions d'interactions humaines. Québec: Les Presses de l'Université Laval. 
Tardif, M. et Ziarko, H. (dir.) (1997). Continuité et ruptures dans la formation des maitres au Québec. Québec: Les Presses de l'Université Laval.

Tilman, F. et Delvaux, É. (2000). Manuel de la formation en alternance. Lyon: Chronique Sociale.

Vanhulle, S., Merhan, F. et Ronveaux, C. (2007). Introduction. Du principe d'alternance aux alternances en formation des adultes et des enseignants : un état de la question. In F. Merhan, C. Ronveaux et S. Vanhulle (dir.), Alternances en formation (p. 7-45). Bruxelles: De Boeck.

Violet, D. (1997). Pour une pratique pragmatique de l'alternance. In D. Violet (dir.), Formation d'enseignants et alternances (p. 15-37). Paris: L'Harmattan.

Voisin, A. (1993). Alternance ou alternances? Approche institutionnelle des formations alternées qualifiantes destinées aux jeunes. Éducation permanente, 115(2), 35-44.

Wittorski, R. (2007). Professionnalisation et développement professionnel. Paris: L'Harmattan. 
